\title{
Effects of geometric and material parameters on band-gaps of piezoelectric vibration energy harvesting plate with local resonators
}

\author{
Liu Ziniu ${ }^{1}$, Chen Zhongsheng ${ }^{1,2, *}$, Zhang Fan $^{1}$, Yeping Xiong ${ }^{2}$ \\ 1 Science and Technology on Integrated Logistics Support Laboratory, National University of Defense Technology, Changsha \\ 410073, P. R. China \\ 2 Faculty of Engineering and the Environment, University of Southampton, UK \\ chenzhongsheng@nudt.edu.cn
}

\begin{abstract}
Continuously powering wireless sensor nodes (WSNs) has been one key problem in structural health monitoring. Piezoelectric energy harvesting (PEH) from environmental vibrations has been a potential way to make low power consumption WSNs self-powered. One kind of vibration energy harvesting plate with local resonators embedded in piezoelectric patches is presented in this paper. Due to its distinct dynamic performances: band gaps, we can control wave propagating for the purpose of broad band vibration harvesting and higher energy conversion efficiency. Distributions and characteristics of band gaps are affected by geometric and material parameters, thus it's necessary to analyze the effects of these key parameters. In this paper, a theoretical calculation method of vibration propagation characteristics is developed based on finite element method (FEM) and the Floquet-Bloch theorem. Then finite element simulations using Comsol software are done to analyze the effects of different parameters. The results show that we can reduce the beginning frequency of the lowest band gap by increasing the length of resonators, while broadening band gaps by raising the filling ratio of the piezoelectric patches. On the other hand, Young modulus is the main factor of material parameters which markedly affects the beginning and cutoff frequency. The results provide useful theoretical guidelines for optimally designing vibration energy harvesting plates in applications.
\end{abstract}

Keywords-piezoelectric energy harvesting; local resonator; band gaps; FEM;Floquet-Bloch theorem

\section{INTRODUCTION}

In structural health monitoring, it has been one key problem to make low energy consumption wireless sensor nodes selfpowered. Energy harvesting $(\mathrm{EH})$ has received considerable attention[1]due to its ability to collect electric power from ambient energy sources. As one of EH technology, piezoelectric energy harvesting can harvest electrical energy from mechanical vibrations based on the direct piezoelectric effect [2]. Piezoelectric energy harvesting is superior to other vibration-to-electricity conversion mechanisms due to large energy density and ease of implementation, so it has been widely studied [3-5].

Various models representing the electromechanical behavior of piezoelectric energy harvesters are proposed, which cover from lumped parameter models [6] to RayleighRitz type approximate distributed-parameter models [7,8]along with analytical distributed-parameter piezoelectric solution attempts [9].Among these models, however, a prominent problem has been neglected. Assumptions that vibration sources have fixed position, concentrated energy, and single direction excitation are oversimplified for real conditions. Structural vibrations are usually variable and often exist in the form of elastic waves. As a result, the simplification of existing methods makes the energy of waves overlooked and lead to a relatively low efficiency of energy harvesting.

To enhance broad band vibration energy harvesting efficiency, one kind of vibration energy harvesting plate has been proposed. It consists of periodic piezoelectric patches equipped with local resonators that act as local absorbers of mechanical vibrations [10-12].The dynamic performances of this structure, particularly shown as band gaps, depend on the geometric and material properties, thus it's necessary to analyze the effects of these key parameters.

The remainder of this paper is organized as follows. In Section 2,the proposed plate configuration is firstly presented, along with the finite element model and calculations of band gaps. Effects of different geometric and material parameters are then analyzed through variable controlling method in Section 3. Finally, the conclusions of this work are summarized in Section 4.

\section{THREORETICAL CALCULATIONS OF BAND GAPS}

\section{A. Piezoelectric Energy Harvesting Plate}

Fig. 1 shows a thin rectangular plate as the base structure, which is a typical configuration for analysis and application, as well as a good structure for vibration propagation. Structures of piezoelectric patches bonded to the surfaces of base structures have been extensively studied $[13,14]$, while the present work suggests a new plate structure with square holes filled with piezoelectric patches that support a small mass to form a source of local resonance. A unit cell consists of a piezoelectric patch and a local resonator along with their neighboring base structure, as shown in the right of Fig. 1.

Vibrations of the plate under excitations can propagate along the plane of the plate. Because of the existence of local resonators, some waves can't propagate along the periodic cells within specific frequency bands called the "band gaps". Thus, energies of vibrations are localized in unit cells, which can be

Supported by the National Natural Science foundation of China (Grant No. 51577189) 
converted into electricity through piezoelectric patches and their connected circuits.

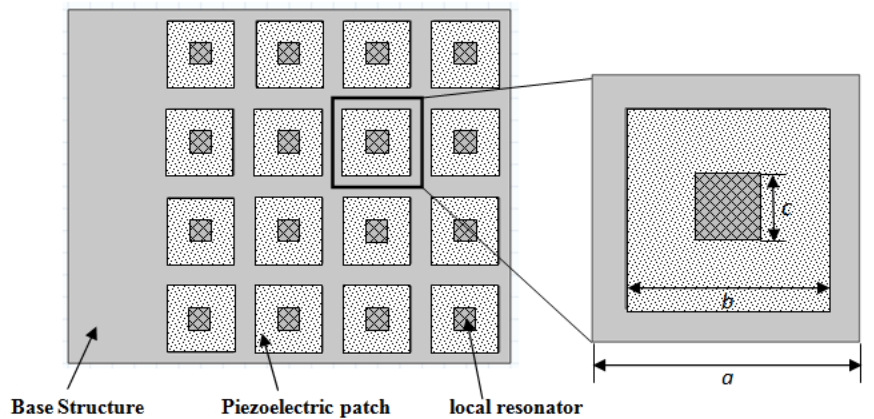

Fig. 1.Schematic diagram of the PEH plate with local resonators.

\section{B. Theoretical Model}

This part builds a theoretical model of the piezoelectric plate based on the finite element method and Floquet-Bloch theorem, which can be available for further calculations.

The equation of harmonic vibrations of plates without considering damping can be expressed as

$$
\left(\mathbf{K}-\omega^{2} \mathbf{M}\right) \mathbf{u}=\mathbf{F}
$$

where $\mathbf{K}$ and $\mathbf{M}$ are stiffness and mass matrices of the whole structure respectively, while $\mathbf{u}$ and $\mathbf{F}$ are generalized displacement and force vectors respectively. And $\omega$ is angular frequency of harmonic vibrations.

The stiffness and mass matrices of the PEH plate, however, are got in a different way from that of ordinary plates, because of the periodic structures of the plate. Due to the discontinuity of materials, different materials should be distributed into different elements in the division of the plate. In this paper, we simplified the model by using the Kirchhoff plate theory firstly. Then divide the plate into quadrilateral finite elements with 3 degrees of freedom (dofs) per node. For each node, the dofs are the transverse displacement $(\omega)$ and the corresponding angular deflections in the $\mathrm{x}$ and $\mathrm{y}$ directions $(\theta \mathrm{x}$ and $\theta \mathrm{y})$.

Calculations of band gaps can be simplified by the FEM and Bloch theorem. Due to the periodicity of the structure, it's only necessary to build the finite element model of one unit cell shown in the right of Fig. 1.

The equation of harmonic vibrations applied to the cells discretized by the FEM can be rewritten as

$$
\left(\mathbf{K}_{\mathrm{c}}-\omega^{2} \mathbf{M}_{\mathrm{c}}\right) \mathbf{u}_{\mathrm{c}}=\mathbf{F}_{\mathrm{c}}
$$

where, $\mathbf{K}_{\mathrm{c}}$ and $\mathbf{M}_{\mathrm{c}}$ are the stiffness and mass matrices of the unit cell respectively, while $\mathbf{u}_{\mathrm{c}}$ and $\mathbf{F}_{\mathrm{c}}$ are the generalized displacement and force vectors respectively.

Fig. 2 shows the displacement degrees of freedom and forces of the unit cell.

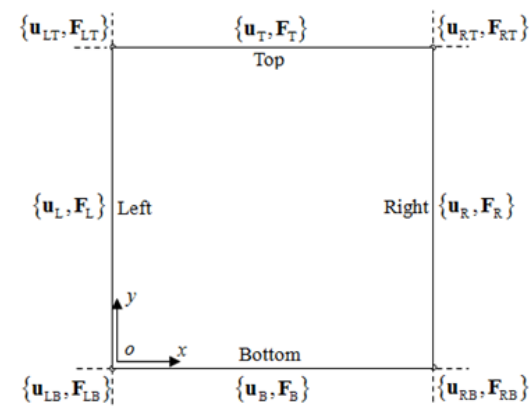

Fig.2. Schematic diagram of displacement degrees of freedom and forces.

In the diagram,

$$
\begin{aligned}
& \mathbf{u}_{\mathrm{c}}=\left[\begin{array}{llllllll}
\mathbf{u}_{\mathrm{B}}^{\mathrm{T}} & \mathbf{u}_{\mathrm{T}}^{\mathrm{T}} & \mathbf{u}_{\mathrm{LB}}^{\mathrm{T}} & \mathbf{u}_{\mathrm{LT}}^{\mathrm{T}} & \mathbf{u}_{\mathrm{RB}}^{\mathrm{T}} & \mathbf{u}_{\mathrm{RT}}^{\mathrm{T}} & \mathbf{u}_{\mathrm{L}}^{\mathrm{T}} & \mathbf{u}_{\mathrm{R}}^{\mathrm{T}}
\end{array}\right]^{\mathrm{T}} \\
& \mathbf{F}_{\mathrm{c}}=\left[\begin{array}{llllllll}
\mathbf{F}_{\mathrm{B}}^{\mathrm{T}} & \mathbf{F}_{\mathrm{T}}^{\mathrm{T}} & \mathbf{F}_{\mathrm{LB}}^{\mathrm{T}} & \mathbf{F}_{\mathrm{LT}}^{\mathrm{T}} & \mathbf{F}_{\mathrm{RB}}^{\mathrm{T}} & \mathbf{F}_{\mathrm{RT}}^{\mathrm{T}} & \mathbf{F}_{\mathrm{L}}^{\mathrm{T}} & \mathbf{F}_{\mathrm{R}}^{\mathrm{T}}
\end{array}\right]^{\mathrm{T}}
\end{aligned}
$$

where subscripts of displacement and force vectors $\mathrm{L}, \mathrm{R}, \mathrm{B}$ and $T$ represent four boundaries of the unit cell, while LB, RB, LT and RT represent its four corners. Based on the Bloch theorem, displacement vectors on the cell's boundaries must compile with the following relations

$$
\begin{aligned}
& \mathbf{u}_{\mathrm{R}}^{\mathrm{T}}=e^{-i k_{x} a_{x}} \mathbf{u}_{\mathrm{L}}^{\mathrm{T}}, \\
& \mathbf{u}_{\mathrm{T}}^{\mathrm{T}}=e^{-i k_{x} a_{x}} \mathbf{u}_{\mathrm{B}}^{\mathrm{T}} .
\end{aligned}
$$

Similarly, displacement vectors on the cell's corners can be expressed by $\mathbf{u}_{\mathrm{LB}}$ as

$$
\begin{gathered}
\mathbf{u}_{\mathrm{RB}}^{\mathrm{T}}=e^{-i k_{x} a_{x}} \mathbf{u}_{\mathrm{LB}}^{\mathrm{T}}, \\
\mathbf{u}_{\mathrm{LT}}^{\mathrm{T}}=e^{-i k_{x} a_{y}} \mathbf{u}_{\mathrm{LB}}^{\mathrm{T}}, \\
\mathbf{u}_{\mathrm{RT}}^{\mathrm{T}}=e^{-i k_{x} a_{x}-i k_{y} a_{y}} \mathbf{u}_{\mathrm{LB}}^{\mathrm{T}} .
\end{gathered}
$$

where, $k_{x}$ and $k_{y}$ are component vectors in $x$ and $y$ directions, while $a_{x}$ and $a_{y}$ represent lattice constants along $x$ and $y$ directions. The former statements can be rewritten in the form of matrix as

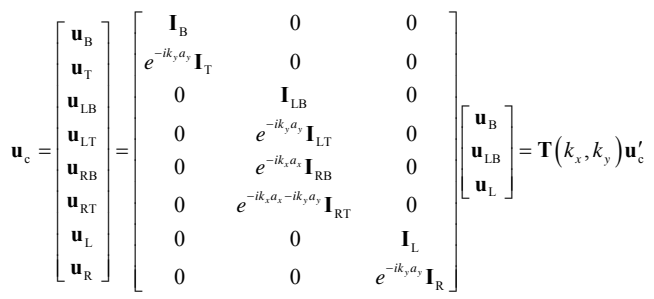

where I is identity matrix, whose subscripts represent its dimensions which match the degrees of nodes on corresponding boundaries. Relations between the force vectors of the unit cell can be acquired through the Bloch theorem and equilibrium conditions. According to relations of four adjacent unit cells shown in the following figure, the equilibrium conditions can be expressed as

$$
\begin{aligned}
\mathbf{F}_{\mathrm{R}}+e^{-i k_{x} a_{x}} \mathbf{F}_{\mathrm{L}} & =0 \\
\mathbf{F}_{\mathrm{T}}+e^{-i k_{x} a_{x}} \mathbf{F}_{\mathrm{B}} & =0
\end{aligned}
$$




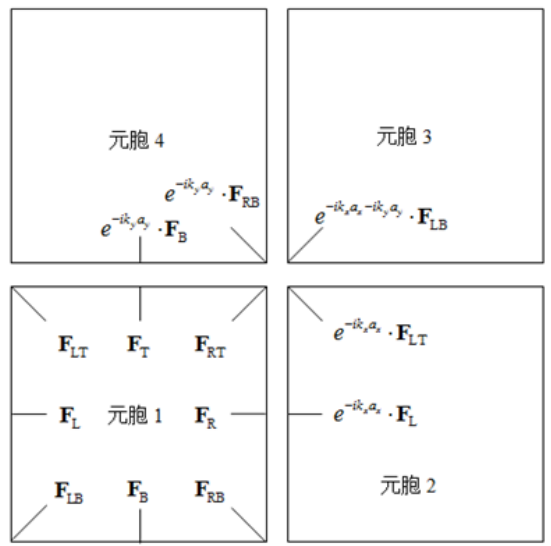

Fig.3. Forces at the boundaries of four connected unit cells.

Meanwhile, the force equilibrium relation of the common point of the four cells is

$$
\mathbf{F}_{\mathrm{RT}}+e^{-i k_{x} a_{x}} \mathbf{F}_{\mathrm{LT}}+e^{-i k_{y} a_{y}} \mathbf{F}_{\mathrm{RB}}+e^{-i k_{x} a_{x}-i k_{y} a_{y}} \mathbf{F}_{\mathrm{LB}}=0
$$

then, substituted in harmonic vibration equation

$$
\left(\mathbf{K}_{\mathrm{c}}-w^{2} \mathbf{M}_{\mathrm{c}}\right) \mathbf{T}\left(k_{x}, k_{y}\right) \mathbf{u}_{\mathrm{c}}^{\prime}=\mathbf{F}_{\mathrm{c}}
$$

Multiply $\mathbf{T}^{\mathrm{H}}\left(\mathrm{k}_{\mathrm{x}}, \mathrm{k}_{\mathrm{y}}\right)$ on both sides of the expression, we have

$$
\mathbf{T}^{\mathrm{H}}\left(k_{x}, k_{y}\right)\left(\mathbf{K}_{\mathrm{c}}-w^{2} \mathbf{M}_{\mathrm{c}}\right) \mathbf{T}\left(k_{x}, k_{y}\right) \mathbf{u}_{\mathrm{c}}^{\prime}=\mathbf{T}^{\mathrm{H}}\left(k_{x}, k_{y}\right) \mathbf{F}_{\mathrm{c}}
$$

The right side can be expanded as

$$
\mathbf{T}^{\mathrm{H}}\left(k_{x}, k_{y}\right) \mathbf{F}_{\mathrm{c}}=\left[\begin{array}{c}
\mathbf{F}_{\mathrm{T}}+e^{-i k_{x} a_{y}} \mathbf{F}_{\mathrm{B}} \\
\mathbf{F}_{\mathrm{RT}}+e^{-i k_{x}, a_{x}} \mathbf{F}_{\mathrm{LT}}+e^{-i k_{y}, a_{y}} \mathbf{F}_{\mathrm{RB}}+e^{-i k_{x}, a_{x}-i k_{y} a_{a}} \mathbf{F}_{\mathrm{LB}} \\
\mathbf{F}_{\mathrm{R}}+e^{-i k_{x}, \mathbf{F}_{\mathrm{L}}}
\end{array}\right]=\left[\begin{array}{l}
0 \\
0 \\
0
\end{array}\right]
$$

Consequently, we turn the problem into a generalized eigenvalue problem

$$
\left[\mathbf{K}_{\mathrm{c}}^{\prime}\left(k_{x}, k_{y}\right)-w^{2} \mathbf{M}_{\mathrm{c}}^{\prime}\left(k_{x}, k_{y}\right)\right] \mathbf{u}_{\mathrm{c}}^{\prime}=0
$$

where

$$
\begin{aligned}
\mathbf{K}_{\mathrm{c}}^{\prime}\left(k_{x}, k_{y}\right) & =\mathbf{T}^{\mathrm{H}}\left(k_{x}, k_{y}\right) \mathbf{K}_{\mathrm{c}} \mathbf{T}\left(k_{x}, k_{y}\right) \\
\mathbf{M}_{\mathrm{c}}^{\prime}\left(k_{x}, k_{y}\right) & =\mathbf{T}^{\mathrm{H}}\left(k_{x}, k_{y}\right) \mathbf{M}_{\mathrm{c}} \mathbf{T}\left(k_{x}, k_{y}\right)
\end{aligned}
$$

represent reduced stiffness and mass matrices.

We can obtain the value of $\omega$ from Equation 17 when the wave vector $\left(k_{x}, k_{y}\right)$ is specified. Generally, the wave vector has been always set along the edges of the irreducible part of the first Brillouin zone ( $\mathrm{M}-\Gamma-\mathrm{X}-\mathrm{M})$ shown in Fig. 4. The solution is a dispersion curve in the $\omega-k$ coordinates. There exist as many curves as there are eigenvalues of the problem in Equation 17. If two neighboring curves do not overlap each other, then there is a gap along the $\omega$ axis in which no wave propagation will occur. This gap between dispersion curves is called the band-gap. The next work of this paper is to calculate and analyze band gaps with the help of Comsol software.

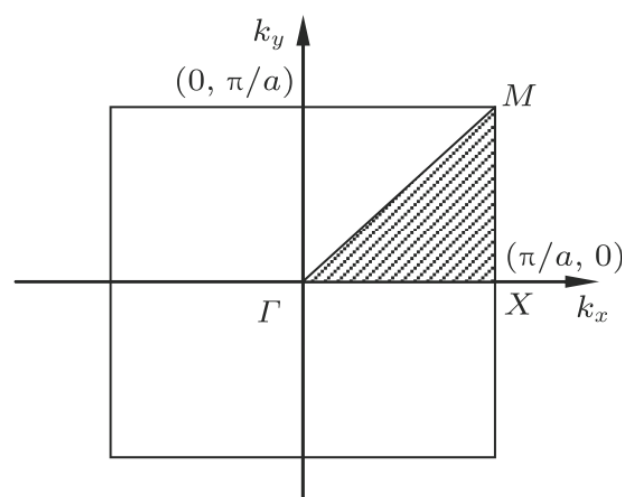

Fig. 4. Schematic diagram of the first Brillouin zone (square) and the irreducible Brillouin zone(triangle area) .

\section{Simulation of Band Gaps}

This part shows an example of finite element simulation for the PEH plate, which ends up with a band structure diagram, displaying the distribution of band gaps. Materials used in simulation for the base, piezoelectric patch and resonator are respectively epoxy resin, polyvinylidene fluoride (PVDF) and lead. Their properties are shown in the following table. The length of the cell $(a)$, the patch $(b)$ and the resonator (c) are first set as $0.04 \mathrm{~m}, 0.03 \mathrm{~m}$ and $0.01 \mathrm{~m}$ respectively.

TABLE I . MATERIALS'PROPERTIES IN SiMULATIONS

\begin{tabular}{|c|c|c|c|c|}
\hline structure & material & $\begin{array}{c}\text { Young } \\
\text { module(GPa) }\end{array}$ & $\begin{array}{c}\text { Poison } \\
\text { ratio v }\end{array}$ & density $\left(\mathbf{k g} / \mathbf{m}^{\mathbf{3}}\right)$ \\
\hline base & epoxy & 0.12 & 0.33 & 1180 \\
\hline patch & PVDF & 31 & 0.36 & 1176 \\
\hline resonator & lead & 17 & 0.42 & 11600 \\
\hline
\end{tabular}

The simulation results in dispersion curves under $14 \mathrm{kHz}$ as shown in Fig. 5 and the shade area represents the lowest band gap. The beginning and cutoff frequency of the lowest band gap are $6 \mathrm{kHz}$ and $9.6 \mathrm{kHz}$ respectively, thus the size of it is $3.6 \mathrm{kHz}$.In this band gap, no dispersion curves occur.Thus energies of waves are localized in unit cells, and can be converted into electricity through piezoelectric patches and their connected circuits.

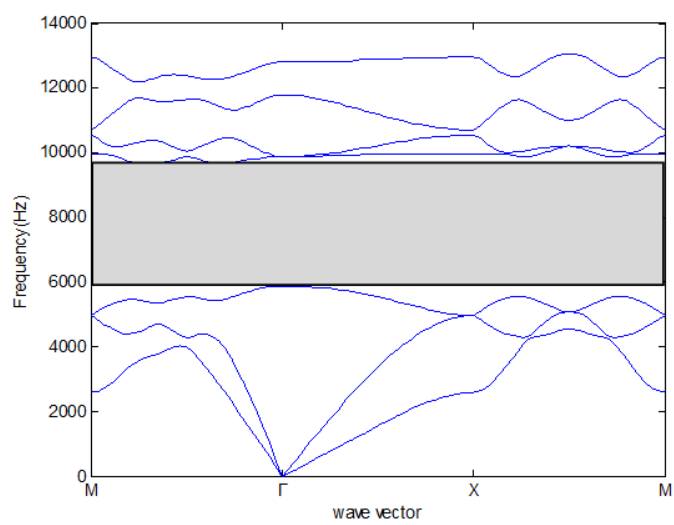

Fig.5. Simulated band gaps in Comsol 


\section{EFFECTS OF GeOMETRIC AND MATERIAL PARAMETERS}

In this section, we use parametric modeling method to analyze the effects of different parameters on band gaps. The effects are mainly shown in two aspects: the beginning frequency and the width of the lowest band gap.

\section{A. Effects of Geometric Parameters}

Assuming the length of the resonator $(c)$ varies from $3 \mathrm{~mm}$ $19 \mathrm{~mm}$ while other parameters remain unchanged, the simulated band gaps show that with the increasing of the parameter $c$, the beginning frequency decreases while the cutoff frequency basically stay unchanged and the size of the band gap becomes larger. Moreover, parameter scanning using Comsol software when wave vector $\left(k_{x}, k_{y}\right)$ is fixed at point $(0,0)$ results in agreement with this conclusion, as shown in Fig. 6. So, we can conclude that adjustment of the length of resonators can help us control the beginning frequency of the lowest band gap while keeping the cutoff frequency unchanged.

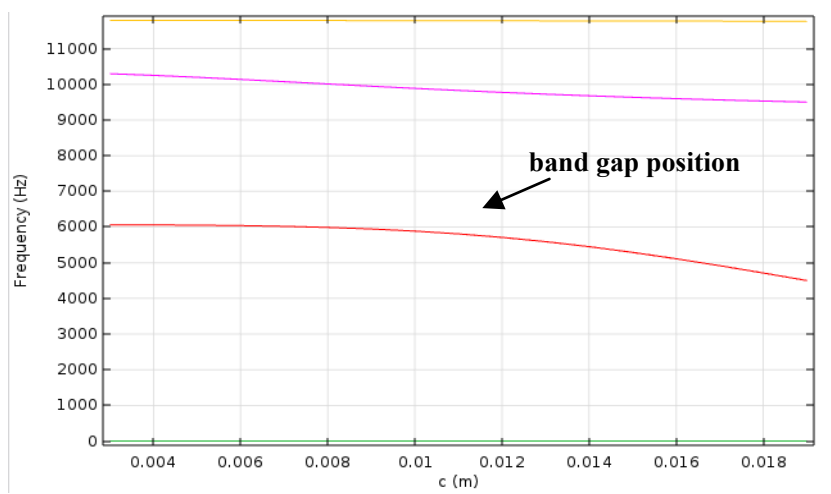

Fig.6.Effects of parameter con the lowest band gap

Similarly, we analyze effects of the parameters $b$ and $a$ on band gaps.

Fig. 7 shows that with the increasing of the variable $b$, both the beginning and cutoff frequency increase. The increasing of the latter is much more apparent compared with that of the former. Consequently, the size of the band gap becomes larger. As a result, we can adjust the cutoff frequency rapidly by controlling the length of patches.

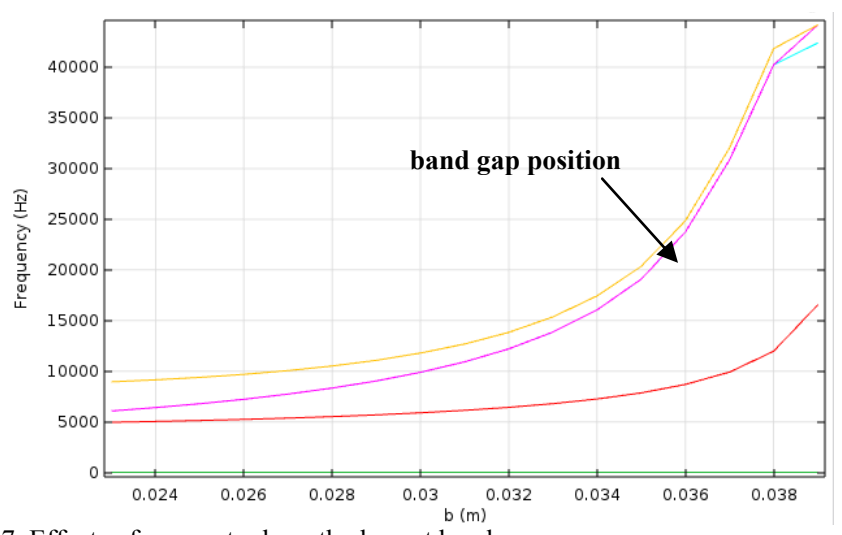

Fig.7. Effects of parameter $b$ on the lowest band gap

On the contrary, as the variable $a$ increases, both the beginning and cutoff frequency decrease. Similarly, the decreasing of the latter is much more apparent compared with that of the former. Thus, the size of the band gap becomes smaller. If we define the ratio of $b$ versus $a$ as the filling ratio of the piezoelectric patch, we can draw a conclusion from Fig. 7 and 8 that this filling ratio has a marked impact on the size of the lowest band gap. With the increasing of this ratio, the size increases significantly.

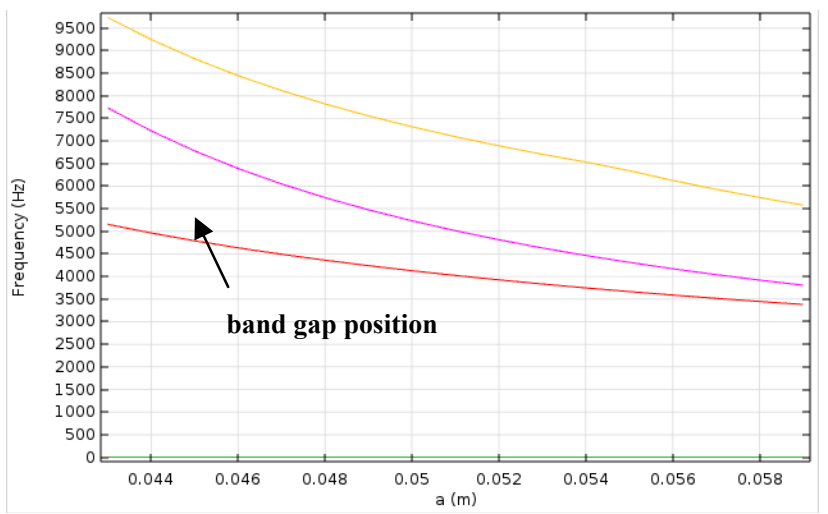

Fig.8. Effects of parameter a on the lowest band gap

\section{B. Effects of Material Parameters}

In order to analyze the effects of materials, we change the material of the base structure at the beginning. As table 2 shows, different base materials have different dispersion curves which vary in frequency distribute ranges, beginning and cutoff frequencies and sizes of band gaps.

TABLE II .PROPERTIES OF MATERIALS FOR COMPARISON

\begin{tabular}{|c|c|c|c|c|}
\hline $\begin{array}{c}\text { Base } \\
\text { material }\end{array}$ & $\begin{array}{c}\text { The fourth } \\
\text { band gap } \\
\text { range (Hz) }\end{array}$ & $\begin{array}{c}\text { Beginning } \\
\text { frequency } \\
\text { (Hz) }\end{array}$ & $\begin{array}{c}\text { Cutoff } \\
\text { frequency } \\
\text { (Hz) }\end{array}$ & $\begin{array}{c}\text { Size of } \\
\text { band } \\
\text { gap (Hz) }\end{array}$ \\
\hline epoxy & $9600 \sim 10000$ & 6000 & 9600 & 3600 \\
\hline aluminum & $(4.8 \sim 6) \times 10^{4}$ & $4.8 \times 10^{4}$ & $4.8 \times 10^{4}$ & 0 \\
\hline rubber & $2500 \sim 3000$ & 2400 & 2500 & 100 \\
\hline
\end{tabular}

For the purpose of making a more clear understanding, keep the base and resonator materials unchanged and calculate band gaps of different material parameters of the piezoelectric patch. We only choose the piezoelectric patch to study because its properties are not fixed and change easily with different experimental and ambient conditions.

Fig. 9 shows the effects of the Young module (E)of the piezoelectric patch on the lowest band gap through parameter scanning, where figure a shows the result of epoxy-base and figure $b$ shows the result of aluminum-base. 


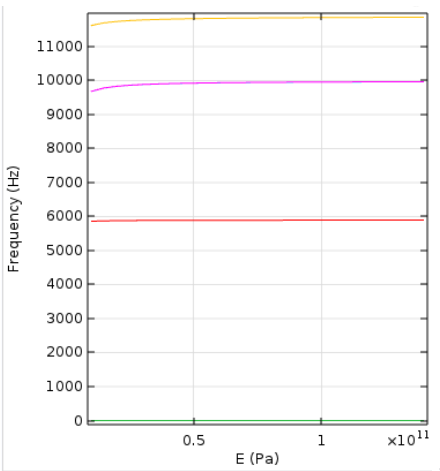

(a)

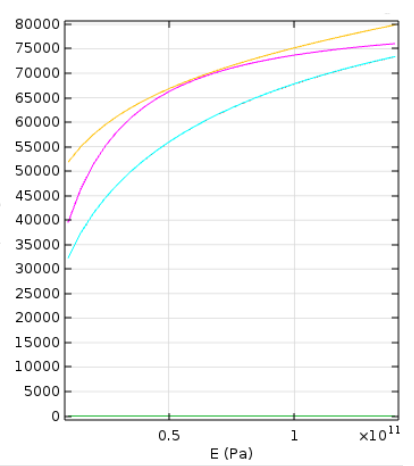

(b)
Fig.9. Effects of Young module on the lowest band gap

From comparison of the two pictures, we can see that the trends of frequencies are completely different due to different base materials. Fig. 9(a) shows that the beginning and cutoff frequency basically remain unchanged when the Young module changes, while Fig. 9(b) shows a uptrend with the increasing of the Young module.

The research of Hirsekorn[15] illustrates the mechanism of band gaps of locally resonance and simplifies the unit cell to a mass-spring lumped parameter system. The beginning and cutoff frequency are evaluated by analyzing resonance mode and frequency of this simplified system. Suppose that $m_{1}$ and $m_{2}$ represent respectively the effective mass of the resonator and base in a unit cell, and $k$ represents the effective stiffness of the coat. At the beginning frequency, the mass $m_{1}$ resonates under the spring $k$ as shown in Fig. 11(a); on the other hand, at the cutoff frequency, the mass $m_{1}$ and $m_{2}$ resonates under the connection of the spring $k$. The dotted line shows the position of the unmoved point.
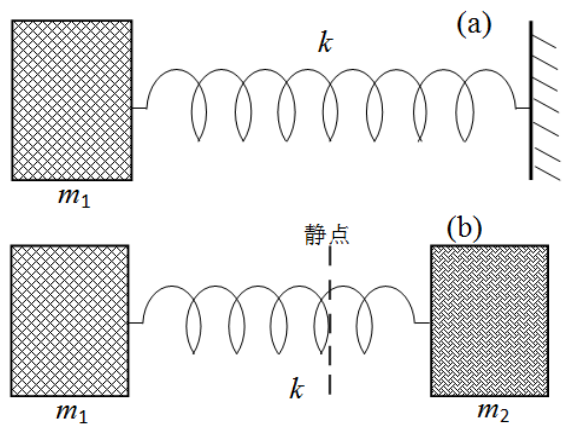

Fig. 10. Simplified mass-spring model

The beginning frequency evaluated from resonator shown in Fig. 10(a) is

$$
f_{1}=\frac{1}{2 \pi} \sqrt{\frac{k}{m_{1}}}
$$

The cutoff frequency evaluated from resonator shown in Fig. 10(b) is

$$
f_{2}=\frac{1}{2 \pi} \sqrt{\frac{k\left(m_{1}+m_{2}\right)}{m_{1} m_{2}}}
$$

One reasonable explanation of the two different phenomena shown in Fig. 9 can be found in the formula of effective stiffness of series springs

$$
k=\frac{k_{1} k_{2}}{k_{1}+k_{2}}=1 /\left(\frac{1}{k_{1}}+\frac{1}{k_{2}}\right)
$$

where $k_{1}$ and $k_{2}$ represent the coefficient of elasticity of the base and patch materials, which are proportional to the Young module of the materials.

Epoxy's Young module is $0.12 \mathrm{Gpa}$, while piezoelectric patch is $31 \mathrm{Gpa}$, so that the corresponding stiffness $k_{1} \ll k_{2}$ From the formula above, we can get that $k_{1} \approx k_{2}$, that is to say the effective stiffness basically stay unchanged no matter how patch's Young module changes, thus a unchanged eigen frequency as displayed in the left of Fig. 9. On the contrary, aluminum's Young module is 69Gpa, which is at the same order of magnitude compared with that of patch. From the formula, we can conclude that with $k_{1}$ unchanged and $k_{2}$ increasing, the effective stiffness increases and thus a increasing of the beginning and cutoff frequency.

\section{CONCLUSIONS}

In this paper, a theoretical calculation method of vibration propagation characteristics is developed based on finite element method (FEM) and Floquet-Bloch theorem. Then finite element simulations using Comsol software are done to calculate the band gaps of the PEH plate. The result shows that energy of propagating waves is indeed localized because of the locally resonance structures.

Analysis of the effects of different parameters using variable controlling method is then presented. The results show that size of resonator mainly affects the beginning frequency of the lowest band gap, while filling ratio of piezoelectric patch has a marked impact on the size of it. On the other hand, the Young module is the main factor of material parameters which markedly affects the beginning and cutoff frequency. A reasonable explanation using the effective stiffness of series springs is proposed for two different frequency curves of different base materials.

In order to control wave propagation better and improve the efficiency of PEH plates, further experimental verifications and deeper studies are needed for comprehensive understanding of band gaps and their influence factors.

\section{REFERENCES}

[1] A.Erturk and D.J.Inman, Piezoelectric Energy Harvesting. John Wiley \& Sons, 2011.

[2] H.S.Kim, J.H.Kim,and J.Kim, "A review of piezoelectricenergy harvesting based on vibration,"International journal of precision engineering and manufacturing,vol. 12,no. 6, pp. 1129-1141, December 2011.

[3] H.A.Sodano, D.J.Inman, and G.Park, “A review of powerharvesting from vibration using piezoelectric materials,"Shock and Vibration Digest, vol.36, no. 3, pp. 197-205, May 2004.

[4] S.R.Anton and H.A.Sodano, "A review of power harvestingusing piezoelectric materials (2003-2006),"Smart materials and Structures,vol. 16,no. 3, pp. R1, May 2007.

[5] F.Lu, H.P.Lee, and S.P.Lim,"Modeling and analysis ofmicro piezoelectric power generators for micro-electromechanical-systems 
applications,"Smart Materials and Structures, vol. 13, no. 1, pp.57-63, November 2003.

[6] H.A.Sodano, G.Park, and D.Inman, "Estimation of electriccharge output for piezoelectric energy harvesting," Strain, vol. 40,no. 2, pp. $49-58$, May 2004.

[7] M. Kim, J. Dugundji, and B.L. Wardle,"Effect of electrodeconfigurations on piezoelectric vibration energy harvestingperformance,"Smart Materials and Structures,vol. 24, no. 4, p.045026, March 2015.

[8] N.E. Dutoit and B.L. Wardle,"Experimental verification ofmodels for microfabricated piezoelectric vibration energyharvesters,"AIAA journal,vol. 45, no.5,pp.1126-1137, May 2007.

[9] N.E. Dutoit, B.L. Wardle, and S.G. Kim, "Design considerations fo MEMS-scale piezoelectric mechanical vibration energy harvesters,'Integrated Ferroelectrics,vol. 71,pp.121-160, Febuarary 2005.

[10] K.M. Ho, C.K. Cheng, Z. Yang,"Broadband locally resonant sonic shields,"Broadband locally resonant sonic shields, vol. 83, no. 26, pp. 5566-5568, November 2003.
[11] J.Sanchez-dehesa, V.M.Garcia-chocano, D. Torrent, F. Cervera, S. Cabrera, and F. Simon,"Noise control by sonic crystal barriers made of recycled materials,"Journal of the Acoustical Society of America., vol. 129, pp. 1173-1183, March 2011.

[12] V.Romero-garcia, J.V.Sanchez-perez,and L.M.Garcia-raffi, “Tunable wideband bandstop acoustic filter basedon two-dimensional multiphysical phenomena periodic systems,"Journalof Applied Physics, vol. 110, p.014904, July 2011.

[13] S.B. Chen, G. Wang, "Wave propagation in beams with anti-symmetric piezoelectric shunting arrays,"Chinese Physics B, vol. 25, no. 3, pp. 034301, Jan. 2016.

[14] I.C. Lien and Y.C. Shu, "Array of piezoelectric energy harvesting by the equivalent impedance approach,"Smart Materials and Structures, vol. 21, pp. 082001, July 2012.

[15] M. Hirsekorn, "Small-size sonic crystals with strong attenuation bands in the audible frequency range,"Applied Physics Letters, vol. 84, pp. 3364-3366, March 2004. 